\title{
Plans for exotic bottomonium-like states at Belle II
}

\author{
Umberto Tamponi* \\ INFN - Sezione di Torino \\ E-mail: tamponi@to.infn.it
}

\begin{abstract}
Large mass, non-relativistic dynamics, large energy level spacing and clear experimental signature are few of the unique characteristics of bottomonia that make their sector rich with a wide range of opportunities of study, from the the spin structure of QCD to the extensions of the standard model Lagrangian, from the non- $q \bar{q}$ states and light quarks dynamics to the gluon fragmentation functions. Experimentally, only high-luminosity $e^{+} e^{-}$colliders with $\sqrt{s} \approx 10 \mathrm{GeV}$ can collect enough statistics to study this system in details and fully exploit its potential. For this reason the Belle II experiment at Super-KEKB collider offers the most promising prospects for the study of bottomonia in the next decade. We will here review the opportunities offered by data taking periods at the $\Upsilon(3 S), \Upsilon(5 S)$ and $\Upsilon(6 S)$ energies, focusing on the variety of studies that can be conducted using bottomonium annihilations: study of conventional and exotic quarkonium spectroscopy, the search for new physics in rare decays of heavy mesons, the study of the light scalar meson family using di-pion transitions among bottomonia, and study of QCD bound states like deuteron and di-baryons with astrophysics implications.
\end{abstract}

XVII International Conference on Hadron Spectroscopy and Structure - Hadron 2017

25-29 September, 2017

University of Salamanca, Salamanca, Spain

${ }^{*}$ Speaker. 


\section{QCD: new frontiers within the standard model}

The experiments at the B-factories left an incredibly rich legacy in many the sectors of particle physics, from the electroweak one, with the observation of the $\mathrm{CP}$ violation in $B \bar{B}$ mesons systems, to the searches for new physics, with stringent upper limits on new gauge bosons [1]. Perhaps the most surprisingly rich output came from the study of the quarkonia. Since long this system is regarded as a promising gateway to tackle the elusive phenomenology connected to the non-perturbative regime of quantum chromo-dynamics [2]. This sector of the standard model is still now, more than forty years after its formalization, difficult to threat theoretically and rich of unexpected discoveries from the experimental side: overall, the phenomenology of the quark interactions is far from being understood [3]. Each manifestation of the non-perturbative QCD is indeed treated with a different effective theory approach, more or less directly related with the core QCD lagrangian. At the B-factories two, and perhaps more in future, of these manifestations can be investigated: the QCD of bound states through heavy and light hadron spectroscopy, including hyperons and charmed baryons, and quark and gluon fragmentation though the inclusive production of hadrons in $e^{+} e^{-} \rightarrow q \bar{q}$ and $e^{+} e^{-} \rightarrow \Upsilon(n S) \rightarrow g g g$ events. In this review we will sketch the impact on these sectors of a data taking at the $\Upsilon(3 S), \Upsilon(5 S)$ and $\Upsilon(6 S)$ energies with the Belle II experiment.

\section{The BelleII experiment and its potential}

The Belle II experiment [4] at the Super-KEKB $e^{+} e^{-}$collider [5] is currently the only experiment with full capability to explore the exotica and the QCD phenomenology in the bottomonium sector. While valuable informations about production and, with severe limitations, spectroscopy of narrow bottomonia can came from the LHC-based experiments, an high-energy hadronic collision is not suitable to study final state without an $\Upsilon(1 S, 2 S, 3 S)^{1}$, transitions with emission of neutral mesons, decays of quarkonia in multi-hadronic final states and the whole phenomenology of the states located above the open flavor thresholds. Given the existing samples and the main physics goal of Belle II, which remains the search for new physics in rare $B$ meson decays, a bottomonium program intended to maximize the physics output with as little data as possible has been prepared, and is sketched in Table 1.

Belle II is scheduled to start the data taking in the spring 2018, and collect $50 \mathrm{ab}^{-1}$ at the $\Upsilon(4 S)$ energy. With respect its predecessor, the Belle Experiment, Belle II will have larger acceptance, improved tracking efficiency and resolution, and a better particle identification. An improved calorimeter readout and clustering algorithm have been implemented to compensate the increased backgrounds. The experiment is articulated over three phases: Phase I, concluded in 2017, consisted in the first commissioning of the Super-KEKB complex, for which the Belle II detector was replaced by the Beast II commissioning detector [7]. Phase II will span from January to the summer 2018: during this phase the final component of the accelerator will be commissioned, including the final focusing magnets, and the Belle II detector, with the silicon vertex detector replaced by the Beast II (Phase II configuration) apparatus for the beam background measurement, will take the

\footnotetext{
${ }^{1}$ With few notable exceptions [6], both Atlas, CMS and LHCb mostly rely on di-muon triggers for their quarkonium physics program, making them sensitive almost only to final states involving a narrow vector state.
} 
Table 1: Summary of the samples collected by the first generation of B-factories, together with the Belle II proposal. The numbers reported are luminosities in $\mathrm{fb}^{-1}$, while the corresponding number for bottomonium decays is reported in brackets. In the last column we report the fraction of non- $(4 S)$ samples with respect the $\Upsilon(4 S)$ one.

\begin{tabular}{lccccccc}
\hline \hline Experiment & $\Upsilon(1 S)$ & $\Upsilon(2 S)$ & $\Upsilon(3 S)$ & $\Upsilon(4 S)$ & $\Upsilon(5 S)$ & $\Upsilon(6 S)$ & $\Upsilon \Upsilon(n S)$ \\
\hline CLEO & $1.2(21)$ & $1.2(10)$ & $1.2(5)$ & $16(17.1)$ & $0.1(0.4)$ & - & $23 \%$ \\
BaBar & - & $14(99)$ & $30(122)$ & $433(471)$ & $\mathrm{R}_{b}$ scan & $\mathrm{R}_{b}$ scan & $11 \%$ \\
Belle & $6(102)$ & $25(158)$ & $3(12)$ & $711(772)$ & $121(36)$ & 5.5 & $23 \%$ \\
\hline BelleII & - & - & $300(1200)$ & $5 \times 10^{4}\left(5.4 \times 10^{4}\right)$ & $1000(300)$ & $100+400(\mathrm{scan})$ & $3.6 \%$ \\
\hline \hline
\end{tabular}

first collision data. During this phase is unlikely to have more than $40 \mathrm{fb}^{-1}$ at the $\Upsilon(4 S)$ energy, making any bottomonium physics unlikely. Finally, starting from January 2019, Phase III will start with the full detector.

\subsection{Hunt for exotica and physics at the thresholds: $\Upsilon(5 S)$ and $\Upsilon(6 S)$}

While in the charmonium sector many exotica are known, only two states have been observed in bottomonium, closely related to each other: $Z_{b}(10610)^{ \pm}$and $Z_{b}(10650)^{ \pm}$[8]. For the former, the neutral partner have been observed as well [9]. Despite the lack of other experimental observation, a rich spectrum of states is predicted by all the effective theories used to model the light-quark contributions to the heavy meson spectra, like the tetraquark model and the molecular model [10, 11]. In a recent proposal for a data taking between the $\Upsilon(5 S)$ and $\Upsilon(6 S)$ energies, the authors count twelve molecular states in the region where the $B \bar{B}, B^{\star} \bar{B}^{\star}$ and $B^{\star} \bar{B}^{\star}$ open up [12]. The investigation of these new states is experimentally challenging since they can be produced only by hadronic or radiative transitions from higher vector states, predicted in the primary $e^{+} e^{-}$collision. In either case, running at the highest possible center of mass energy is preferable, possibly even above 11.4 $\mathrm{GeV}$. However, in the current configuration the Super-KEKB complex can deliver $e^{+} e^{-}$collision with energy in the center of mass just above the $\Upsilon(6 S)$ mass, at $11.02 \mathrm{GeV} / \mathrm{c}^{2}$. Given this limitation, the most reasonable energy to take data at the the $\Upsilon(6 S)$ peak where the hadronic cross section is maximum. This energy is below the threshold for the hadronic production of most of the predicted exotica, leaving the radiative decay the only mean to access these states [14]. This fact makes the direct search for these states particularly challenging, since the radiative transitions are expected to be much smaller than the hadronic ones. Up to now no radiative decay of the any bottomonium state above threshold has been observed. The only search for bottomonium exotica in radiative decay conducted by Belle, that searched for the bottomonium counterpart of the $X(3872)$ in $\Upsilon(5 S)$ radiative decay, was inconclusive [15].

However, another option that should be explored is the search of these exotica in deviation from the expected properties of hadronic transitions between known, conventional states. It has been recently pointed out that the $Z_{b}$ can modify the mass spectrum of dipion transitions like $\Upsilon(4 S) \rightarrow \pi^{+} \pi^{-} \Upsilon(1 S)$ [16] or even $\Upsilon(3 S) \rightarrow \pi^{+} \pi^{-} \Upsilon(1 S)$ [17], de-facto opening the field of the 
Table 2: Summary of the exotic states in the bottomonium sector, from the molecular and the tetraquark model. The table is adapted from [12] and [13].

\begin{tabular}{lccc}
\hline \hline State & I & $J^{P C}$ & production mode [threshold] \\
\hline \hline$Z_{b}$ & 1 & $1^{+}$ & $\pi[10.75]$ \\
$Z_{b}^{\prime}$ & 1 & $1^{+}$ & $\rho[10.79]$ \\
$W_{b 0}$ & 1 & $0^{+}$ & $\rho[11.34], \gamma[10.56]$ \\
$W_{b 0}^{\prime}$ & 1 & $0^{+}$ & $\rho[11.43], \gamma[10.65]$ \\
$W_{b 1}$ & 1 & $1^{+}$ & $\rho[11.38], \gamma[10.61]$ \\
$W_{b 2}$ & 1 & $2^{+}$ & $\rho[11.43], \gamma[10.65]$ \\
$X_{b 1}$ & 0 & $1^{+}$ & $\eta[11.15]$ \\
$X_{b 1}^{\prime}$ & 0 & $1^{+}$ & $\eta[11.20]$ \\
$X_{b 0}$ & 0 & $0^{+}$ & $\omega[11.34], \gamma[10.56]$ \\
$X_{b 0}^{\prime}$ & 0 & $0^{+}$ & $\omega[11.43], \gamma[10.65]$ \\
$X_{b}$ & 0 & $1^{+}$ & $\omega[11.39], \gamma[10.61]$ \\
$X_{b 2}$ & 0 & $2^{+}$ & $\omega[11.43], \gamma[10.65]$ \\
$Y_{1}$ & 0 & $1^{-}$ & $e^{+} e^{-}$direct $[10.89]$ \\
$Y_{2}$ & 0 & $1^{-}$ & $e^{+} e^{-}$direct $[10.90]$ \\
$Y_{3}$ & 0 & $1^{-}$ & $e^{+} e^{-}$direct $[10.98]$ \\
$Y_{4}$ & 0 & $1^{-}$ & $e^{+} e^{-}$direct $[11.14]$ \\
$Y_{5}$ & 0 & $1^{-}$ & $e^{+} e^{-}$direct $[13.04]$ \\
\hline \hline
\end{tabular}

search of exotica in virtual loops ${ }^{2}$. The study of hadronic transitions can also shed light onto the spectrum of conventional quarkonia. Several narrow states which are still missing can be reached via hadronic transitions from the $\Upsilon(6 S): \Upsilon_{J}(2 D), \eta_{b 2}(1 D), h_{b}(3 P), h_{b 3}(1 F), \Upsilon_{J}(2 D)$ are all reachable with $\pi \pi, \eta$ or $\omega$ transitions from the $\Upsilon(6 S)$. Theoretical predictions on the branching fractions for these transitions are not available yet. The study of the hadronic transitions, in particularly the di-pion one, offers also a new opportunities in the low meson sector. Transitions with large $\mathrm{Q}$ value such $\Upsilon(4 S, 5 S, 6 S) \rightarrow \pi \pi \Upsilon(1 S, 2 S, 3 S)$ can get sizable contribution by scalar meson states. The $f_{0}$ has been already observed to be the dominant contributor to $\Upsilon(4 S) \rightarrow \pi \pi \Upsilon(1 S)$, and in the future, with much higher statistics, one could expect to see contributes from the $f_{2}$ and the $\sigma$ meson as well $[16,20]$.

In addition to the search for exotica with a data taking at fixed energy at the $\Upsilon(6 S)$, another issue that must be addressed by Belle II is the nature of the $\Upsilon(5 S)$ and $\Upsilon(6 S)$ themselves and the general structure of the hadronic cross section in the threshold region. Being located at the $B_{s} \bar{B}_{s}$ threshold, the $\Upsilon(5 S)$ could be the admixture of conventional and molecular bottomonium. In addition to that, several vector tetraquark are predicted in the region between the $\Upsilon(5 S)$ and $\Upsilon(6 S)$. On top of these very specific motivations, few generic considerations should be done. First, all the exotica are located around the threshold, so it is natural to scan carefully these region measuring the line-shape of the cross section for both inclusive and exclusive processes. Second, a fine scan

\footnotetext{
${ }^{2}$ Interestingly, the main goal of Belle II is exactly the search for new physics contributions to rare decay as additional diagrams with new virtual loops. Possibly the search for bottomonium exotica could be done in the very same way, looking at well known, experimentally pure transitions.
} 
of the same region in charmonia showed totally unexpected results: the famous $Y(4260)$ seems now to be a superimposition of two states, rather than a single one [18]. Third, the Belle scan of $\Upsilon(5 S)$ region showed inconclusive results: even if it allowed to exclude the presence of narrow resonances, the analysis of the cross section highlighted a complex interplay between the threshold in the continuum and the bottomonium resonance [19]. For these reasons a high statistics, fine scan of the region spanning from the $\Upsilon(4 S)$ to the $\Upsilon(6 S)$ has been proposed [12].

\subsection{Precision QCD, dark matter and hyperon spectroscopy: $\Upsilon(3 S)$ and $\Upsilon(4 S)$}

Before moving to a brief description of the physics program below the threshold, we would like to point out a precision QCD measurement on conventional quarkonium that can be done at Belle II using the $\Upsilon(4 S)$ and $\Upsilon(5 S)$ datasets. Tagging the dacay cascades $\Upsilon(5 S) \rightarrow \pi \pi h_{b}(1 P) \rightarrow$ $\pi \pi \gamma \eta_{b}(1 S)$ and $\Upsilon(4 S) \rightarrow \eta h_{b}(1 P) \rightarrow \eta \gamma \eta_{b}(1 S)$, Belle II will be able to both measure with extreme precision the mass and the width of this $s^{3} e^{3}$, for which new, precise NRQCD predictions have been recently released [21], and to explore its decay. Among these, we wish to highly $\eta_{b}(1 S) \rightarrow \gamma \gamma$, which is theoretically well known but has been always an extremely challenging measurement for the experimentalists using the radiative decays $\Upsilon(2 S, 3 S) \rightarrow \gamma \eta_{b}(1 S)$, due to the overwhelming background from radiative $e^{+} e^{-} \rightarrow \gamma \gamma$ process. However, with the hadronic transitions discovered by Belle this background can be significantly reduced, and in the full Belle datasets we expect to have few tens of well reconstructed $\eta_{b}(1 S) \rightarrow \gamma \gamma$ candidates.

In the final part of this work, we would like to discuss the physics program at the narrow resonances. This program is intended not only to address the open questions about few rare hadronic transitions, but also includes several low-energy QCD topics, studies looking at the products of the gluon fragmentation in $\Upsilon(n S) \rightarrow g g g$ annihilation, as well as few searches for physics beyond the standard model. Having to focus a narrow-quarkonia physics program on one energy only, the $\Upsilon(3 S)$ is the more suitable. While the physics at $\Upsilon(2 S)$ and $\Upsilon(1 S)$ has been largely explored by BaBar and Belle and their datasets can still provide important results, the BaBar's studies of $\Upsilon(3 S)$ transitions left few important, unresolved points. The two most outstanding ones are the isospinviolating transition $\Upsilon(3 S) \rightarrow \pi^{0} h_{b}(1 P)$, for which an unexpected evidence has been reported [22], and the $\Upsilon(3 S) \rightarrow \eta \Upsilon(1 S)$, which despite the theoretical predictions has not been observed yet [23].

The $\Upsilon(3 S)$ data sample will allow to study rare decays of the $\chi_{b 0}(n P)$, including the ultra-rare process $\chi_{b 0}(2 P) \rightarrow \tau^{+} \tau^{-}$. This process, suppressed in the standard model, is expected to be largely enhanced by the presenc of new scalar particles [24]. The observation of such a process would be an evidence of phyiscs beyond the standard model, and its non-observation would allow to set new limits in unexplored region of the NP parameters' space.

In conclusion, we would like to briefly sketch the possibilities given by the study of the $\Upsilon(3 S)$ annihilations. This process has been not yet studied in details, but it has already shown few peculiar characteristics. First, the CLEO collaboration reported an unexplained enhancement of strangeness production [25], phenomenon well know to the heavy ion community, which causes a large production rate of hyperons. Bottomonium annihilations are therefore preferable over $e^{+} e^{-} \rightarrow q \bar{q}$ and $B$ decays to study the spectrum if these states. The large production rate would allow to study

\footnotetext{
${ }^{3}$ The width of the $\eta_{c}(1 S)$ is still an open problem in charmonium, while in bottomonium a the experimental resolution is much worst than the theoretical uncertainty.
} 
not only the production, but also the the hyperon-hyperon and hyperon-proton correlations: a pilot study made by Belle produced stringent limits on the existence of the $H$ dibaryon [26], but the goal for BelleII would be to extend this study to the correlation function and to the search for long-lived or stable dibaryons [27], using fully reconstructed events with missing energy. Finally, 1.2 Billions of $\Upsilon(3 S)$ annihilations we plan to study with unprecedented precision the spectrum of the anti-deuteron produced in the annihilation [28, 29]. This study will allow to investigate the foundamental mechanism for the production of anti-nuclei in hadronic events, without involving the corrections for final state interaction needed in the heavy ion collisions. The understanding of the $\bar{d}$ production mechanism is a foundamental for the interpretation of the results on the anti-matter content in the cosmic rays [30] expected by the AMS-02 [31] and GAPS [32] collaborations.

\section{References}

[1] A. J. Bevan et al. [BaBar and Belle Collaborations], Eur. Phys. J. C 743026 (2014).

[2] N. Brambilla et al., Eur. Phys. J. C 71, 1534 (2011).

[3] N. Brambilla et al., Eur. Phys. J. C 74, 2981 (2014).

[4] T. Abe et al. [Belle-II Collaboration], arXiv:1011.0352 (2010).

[5] Y. Morita, K. Akai, T. Furuya, A. Kabe, S. Mitsunobu, M. Nishiwaki and S. Takano, Conf. Proc. C 100523, TUPEB011 (2010).

[6] R. Aaij et al. [LHCb Collaboration], Eur. Phys. J. C 75 no.7, 311 (2015).

[7] M. Nayak [Belle-II Collaboration], PoS BEAUTY 2016, 059 (2016).

[8] A. Bondar et al. [Belle Collaboration], Phys. Rev. Lett. 108, 122001 (2012).

[9] P. Krokovny et al. [Belle Collaboration], Phys. Rev. D 88, 052016 (2013).

[10] A. Esposito, A. L. Guerrieri, F. Piccinini, A. Pilloni and A. D. Polosa, Int. J. Mod. Phys. A 30, 1530002 (2015).

[11] R. F. Lebed, R. E. Mitchell and E. S. Swanson, Prog. Part. Nucl. Phys. 93, 143 (2017).

[12] A. E. Bondar, R. V. Mizuk and M. B. Voloshin, Mod. Phys. Lett. A 32, no. 04, 1750025 (2017).

[13] A. Ali, J. S. Lange and S. Stone, Prog. Part. Nucl. Phys. 97123 (2017).

[14] M. B. Voloshin, Phys. Rev. D 84, 031502 (2011).

[15] X. H. He et al. [Belle Collaboration], Phys. Rev. Lett. 113, no. 14, 142001 (2014).

[16] Y. H. Chen, M. Cleven, J. T. Daub, F. K. Guo, C. Hanhart, B. Kubis, U. G. MeiÃßner and B. S. Zou, Phys. Rev. D 95, no. 3, 034022 (2017).

[17] Y. H. Chen, J. T. Daub, F. K. Guo, B. Kubis, U. G. Meĩ̃ßner and B. S. Zou, Phys. Rev. D 93 no.3, 034030 (2016).

[18] M. Ablikim et al. [BESIII Collaboration], Phys. Rev. Lett. 118 no.9, 092001 (2017).

[19] D. Santel et al. [Belle Collaboration], Phys. Rev. D 93 no.1, 011101 (2016).

[20] F. K. Guo, private communication.

[21] F. Feng, Y. Jia and W. L. Sang, arXiv:1707.05758 [hep-ph] (2017). 
[22] J. P. Lees et al. [BaBar Collaboration], Phys. Rev. D 84091101 (2011).

[23] J. P. Lees et al. [BaBar Collaboration], Phys. Rev. D 84, 092003 (2011).

[24] S. Godfrey and H. Logan, Phys. Rev. D 93, 055014 (2016).

[25] R. A. Briere et al. [CLEO Collaboration], Phys. Rev. D 76012005 (2007).

[26] B. H. Kim et al. [Belle Collaboration], Phys. Rev. Lett. 110, no. 22, 222002 (2013).

[27] G. R. Farrar, arXiv:1708.08951 [hep-ph] (2017).

[28] D. M. Asner et al. [CLEO Collaboration], Phys. Rev. D 75, 012009 (2007).

[29] J. P. Lees et al. [BaBar Collaboration], Phys. Rev. D 89, no. 11, 111102 (2014).

[30] T. Aramaki et al., Phys. Rept. 6181 (2016).

[31] R. Battiston [AMS 02 Collaboration], Nucl. Instrum. Meth. A 588, 227 (2008).

[32] C. J. Hailey, New J. Phys. 11, no. 10, 105022 (2009). 